\title{
Age moderates the effect of socioeconomic status on physical activity level among south Korean adults: cross-sectional analysis of nationally representative sample
}

\author{
Harold H. Lee ${ }^{1 *}$, Ashley E. Pérez ${ }^{2}$ and Don Operario ${ }^{3}$
}

\begin{abstract}
Background: In a nationally representative sample of South Korean adults, we investigated the association between socioeconomic status (SES) and physical activity level, and whether this association varied by age group.

Methods: We used data from 5065 subjects aged $\geq 19$ years who participated in the 6th Korea National Health and Nutrition Examination Survey. Weighted logistic regression examined the SES-physical activity association. Using the International Physical Activity Questionnaire short form, physical activity level was categorized into two groups: meeting the guideline ( $\geq 150 \mathrm{~min} /$ week of moderate intensity physical activity) and not meeting the guideline. Annual household income quartile (first quartile = highest income) and education (elementary, middle school, high school, and college graduates) were used as SES indicators. Sociodemographic covariates included in the adjusted models were marital status, town type (rural/urban), dwelling type, perceived health, federal allowance support (yes/no), and working- and sleeping-hours.
\end{abstract}

Results: In unadjusted models, low income and low education were both associated with significantly lower odds of meeting the physical activity guideline. The income-physical activity association was moderated by age group in both unadjusted and adjusted models. Specifically, among those age $<45$ years, those in the third quartile group had $41 \%$ higher odds $(p<0.01$ ) of meeting the physical activity guideline compared with the first quartile group, after adjusting for covariates. In contrast, among those age $\geq 45$ years, those in the third quartile group had 16\% lower odds $(p<0.01)$ of meeting the physical activity guideline compared with the first quartile group, after adjusting for covariates. Furthermore, the education-physical activity association was moderated by age but only in the adjusted model. Specifically, among those age $<45$ years, high school graduates had a $21 \%$ higher odds of meeting the physical activity guideline compared with college graduates $(p=0.08)$. In contrast, among those age $\geq 45$ years, high school graduates had a $23 \%$ lower odds of meeting the physical activity guideline compared with college graduates $(p=0.01)$.

Conclusion: Future policies that aim to address SES-related health disparities in physical activity among adults in South Korea should consider the different patterns of physical activity in accordance with SES and age.

Keywords: Physical activity, Health disparities, South Korea, Noncommunicable disease

\footnotetext{
* Correspondence: hhlee@hsph.harvard.edu

'Department of Social and Behavioral Sciences, Harvard T.H. Chan School of

Public Health, 401 Park Dr., 428F, Boston, MA 02115, USA

Full list of author information is available at the end of the article
}

(c) The Author(s). 2019 Open Access This article is distributed under the terms of the Creative Commons Attribution 4.0 International License (http://creativecommons.org/licenses/by/4.0/) which permits unrestricted use, distribution, and reproduction in any medium, provided you give appropriate credit to the original author(s) and the source, provide a link to the Creative Commons license, and indicate if changes were made. The Creative Commons Public Domain Dedication waiver (http://creativecommons.org/publicdomain/zero/1.0/) applies to the data made available in this article, unless otherwise stated. 


\section{Background}

South Korea's economy grew rapidly over the last 40-45 years, achieving a $7 \%$ growth rate per year in real per capita income [1]. This exceptional economic growth, however, has been accompanied by increasing income and educational inequalities [2, 3]. More recently, there is an emerging concern about socioeconomic disparities in overall mortality as well as disparities in the prevalence of noncommunicable diseases (NCDs) such as diabetes, hypertension, and obesity among South Koreans [4-8].

One of the four major modifiable behavioral risk factors for NCDs, along with diet, smoking, and drinking, is low rate of physical activity, which accounts for 9\% (5.3 million) of premature deaths globally [9]. To curb this global problem, the World Health Organization (WHO) recommended that nations develop physical activity action plans and policies to increase physical activity levels in their populations [10]. Specifically, WHO recommended that adults engage 150 min per week of moderate-intensity physical activity (e.g., brisk walking) or $75 \mathrm{~min}$ per week of vigorous physical activity (e.g., running), or an equivalent combination of moderate- and vigorous-intensity aerobic activity [11]. According to the WHO Global Health Observatory data repository, the proportion of adults meeting this recommendation is lower in high-income compared to low-income countries [12, 13], with most adults from developed countries (e.g., Australia, UK, and US) not meeting the recommendations [14-16]. In this light, among the OECD countries, following Mexico and Costa Rica, South Korea is ranked 3rd in terms of hours spent on working (i.e., 2024 h per week in 2017) [17], which may minimize the time and cognitive energy to engage in health promoting behaviors, including sleeping and physical activity [18, 19]. Furthermore, a corpus of research conducted in highincome countries has shown that socioeconomically disadvantaged populations are even less likely to engage in adequate levels of physical activity compared with those who have greater socioeconomic advantage [20-25].

Given emerging evidence of health disparities in South Korea, as well as the unequal distribution of meeting physical activity guidelines by socioeconomic status (SES) observed in other high-income countries, low SES Korean adults may be at risk for engaging in lower physical activity levels. While a few studies to date reported that low SES is associated with lower participation in physical activity among adolescents [26], the elderly [27], and metabolic syndrome patients [28] in South Korea, to our knowledge, no studies have examined this relationship among healthy South Korean adults (age $\geq 19$ ). To address this gap, the specific objective of the present study was to investigate the associations between two SES indicators, annual household income and education, and physical activity in a nationally representative sample of South Korean adults. We hypothesized that low household income and education status would be associated with lower odds of meeting the WHO physical activity guideline. However, previous literature has reported that physical activity tends to decline as age increases [12]; specifically, a more progressive decline in physical activity is observed after age $45[16,29]$. Therefore, we further explored whether age group (with age 45 as the cut point) moderated the relationship between SES and physical activity participation.

\section{Methods}

\section{Overview}

The present study employed a cross sectional design using nationally representative data from South Korea. A total of 7380 subjects participated in the 6th Korea National Health and Nutrition Examination Survey (KNHANES VI; 2015) conducted by the Korean Centers for Disease Control and Prevention (KCDC) and the Ministry of Health and Welfare. A full description of the KNHANES methodology has been presented elsewhere $[30,31]$. Briefly, KNHANES is a nationally representative sample of non-institutionalized South Koreans. In order to recruit a representative sample, KNHANES utilized a multi-stage clustered probability design based on administrative district, place of residence, and residential means (i.e. apartment, other than apartment). Participants completed in-person health interviews and health examinations in mobile examination centers with trained staff members. From the total 7380 subjects, we excluded 2315 participants for the following reasons: missing weight variables, aged below 19 years old, and did not completed the International Physical Activity Questionnaire (IPAQ) (Fig. 1). Our analytic sample included the remaining 5065 subjects who completed the IPAQ. However, some covariates had missing values: 'Household

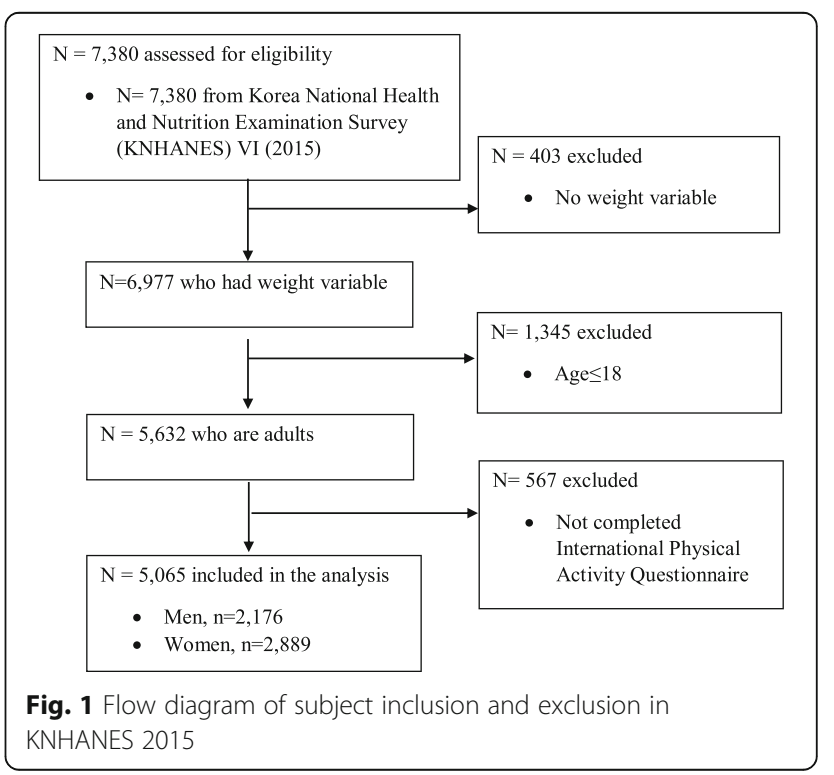


income' was missing among 22 subjects (0.7\%), 'Education' was missing among 9 subjects (0.1\%), 'Federal allowance support' was missing among 4 subjects (0.2\%), 'Sleeping hours' was missing among 74 subjects (1.4\%), 'Perceived health' was missing among 2 subjects (0.01\%), and 'Working hours' was missing among 1642 subjects (32.4\%). We considered removing working hours given the high proportion of missing data, but working hours is a plausible confounder of the SES-PA link so we decided to include it. Overall, relative to those who provided IPAQ data, those who did not were more frequently married, older, and had a lower education $(p<0.05)$.

\section{Measures}

In accordance with other studies on SES gradients in health [32], we examined two self-reported SES indicators included in the KNHANES: annual household income and education. Household income was assessed by dividing monthly household income by the square root of the number of household members (adjusting for sex and each 5-year age stratum), and participants were categorized into four quartile household income groups (upper, moderate, moderate-low, and low). Education was assessed by the question, "What is the highest diploma you obtained from school?" (categories included college graduate, high school graduate, middle school graduate, and elementary school graduate). The primary dependent variable used for this analysis was total volume of physical activity, which was computed using the IPAQ short form. The 9-item IPAQ questionnaire assesses the amount of time spent engaging in five-levels of weekly physical activity: 1) vigorous physical activity spent during work, 2) moderate physical activity spent during work, 3) vigorous physical activity spent during leisure time, 4) moderate physical activity spent during leisure time, and 5) moderate physical activity spent on transportation. To calculate total volume of moderate intensity physical activity, KNHANES staff members doubled the time spent in vigorous physical activity (e.g., $30 \mathrm{~min}$ of vigorous physical activity $=60 \mathrm{~min}$ of moderate intensity physical activity). Then, all minutes were added. KNHANES staff then categorized subjects into two groups in a variable called 'pa_aerobic': those who do not meet the WHO physical activity guideline $(\leq 150 \mathrm{~min}$ per week of moderate-intensity physical activity) and those who meet the physical activity guideline ( $>150 \mathrm{~min}$ per week of moderate-intensity physical activity) [11]. The Korean version of the short-form IPAQ employed in KNHANES has been shown to be valid and reliable [33, 34]. Additional covariates included marital status, town type (rural/urban), dwelling type, perceived health, federal allowance support (yes/no), and working- and sleeping-hours.

\section{Analytic strategy}

All analyses were conducted using R [35], and weights were applied using the svy command. First, we conducted descriptive analyses to examine distributions for all key variables; weighted percentages are reported here. Second, we examined bivariate associations between SES indicators and our primary dependent variable, physical activity. Third, we employed weighted logistic regressions to examine the association between each socioeconomic factor and physical activity level, adjusting for sociodemographic covariates including marital status, town type (rural/urban), dwelling type, perceived health, federal allowance support (yes/no), and working- and sleeping-hours. Given limited research on SES and physical activity in South Korea, covariates were selected as potentially intermediary or confounding variables rather than based on prior literature. Finally, we examined interactions between SES and age group (age $<45$ vs. age $\geq 45$ ), adjusting for sociodemographic covariates.

\section{Results}

Sociodemographic characteristics of the 5065 participants are shown in Table 1 (unweighted and weighted percentages). The mean age was $43.6(\mathrm{SE}=0.4)$ years . About one-quarter of adults aged 19 and older, 76.4\% were married, and $75.1 \%$ had graduated high school. Overall, $49.0 \%$ were classified as not meeting the WHO physical activity guideline.

Bivariate associations of SES and physical activity by age and gender are illustrated in Table 2. Weighted Chi-square statistics showed that the probability of meeting the physical activity guideline significantly differed by income and education. With regard to income, the proportion of those meeting the physical activity guideline was comparable at upper (54.7\%), moderate (52.8\%), and moderate-low $(50.0 \%)$ income levels, but lower among those in the low income group (41.1\%), $\mathrm{F}(1,3)=34.10, p<0.001$. With regard to education, the proportion of those meeting the physical activity guideline was comparable for college graduates (55.7\%) and high school graduates (55.7\%), lower among middle school graduates (43.2\%), and lowest among those who completed elementary school or below (33.5\%), F (1, $3)=7.20, p<0.001$.

The unadjusted and adjusted weighted logistic regressions testing associations of SES and SES*age on physical activity are shown in Table 3. In the unadjusted model, education and income were both significant predictors of meeting the physical activity guideline. Specifically, compared to the upper household income group, the moderate-low- (OR = 0.83, 95\% CI: 0.69-0.99) and low household income groups $(\mathrm{OR}=0.58,95 \%$ CI: $0.47-0.71)$ had significantly lower odds of meeting the physical activity guideline. Additionally, compared to college graduates, middle school $(\mathrm{OR}=0.61,95 \%$ CI: $0.48-0.71)$ and elementary school graduates $(\mathrm{OR}=0.40$, 95\% CI: 0.33-0.49) had significantly lower odds of meeting the physical activity guideline. Age was a significant predictor of meeting the physical activity guideline in the unadjusted model. Specifically, older adults (age $\geq 45$ years) had 
Table 1 Population-Weighted Characteristics of the KNHANES VI Study Sample $(n=5065)$

\begin{tabular}{|c|c|}
\hline & n (weighted \%) \\
\hline Age, mean (SE) & $43.6 \mathrm{yr}(0.38)$ \\
\hline Age $\geq 45$ years & $3269(52.5 \%)$ \\
\hline Female & $2889(51.4 \%)$ \\
\hline Married & $4240(76.4 \%)$ \\
\hline Federal allowance support & $4656(92.5 \%)$ \\
\hline Working hours, mean (SE) & $41.20 \mathrm{~h} / \mathrm{wk} .(0.39)$ \\
\hline Sleeping hours, mean (SE) & $6.76 \mathrm{~h} / \mathrm{d}(0.02)$ \\
\hline \multicolumn{2}{|l|}{ Town type } \\
\hline Dong (i.e., Urban) & 4113 (83.6\%) \\
\hline Ub or myun (i.e., Rural) & $948(16.4 \%)$ \\
\hline \multicolumn{2}{|l|}{ Dwelling } \\
\hline Single house & $1860(35.6 \%)$ \\
\hline 5-story apartment and higher & $2541(50.2 \%)$ \\
\hline 4-story apartment and lower (> $\left.660 \mathrm{ft}^{2}\right)$ & $362(7.1 \%)$ \\
\hline 4-story apartment and lower $\left(\leq 660 \mathrm{ft}^{2}\right)$ & $226(4.5 \%)$ \\
\hline Other & $76(1.5 \%)$ \\
\hline \multicolumn{2}{|l|}{ Perceived health } \\
\hline Very good & $245(5.4 \%)$ \\
\hline Good & $1241(25.8 \%)$ \\
\hline Normal & $2549(50.5 \%)$ \\
\hline Bad & $812(14.9 \%)$ \\
\hline Very bad & $216(3.4 \%)$ \\
\hline \multicolumn{2}{|l|}{ Household income } \\
\hline 1st quartile & $1464(31.3 \%)$ \\
\hline 2nd quartile & $1381(30.1 \%)$ \\
\hline 3rd quartile & $1240(23.3 \%)$ \\
\hline 4th quartile & $958(15.3 \%)$ \\
\hline \multicolumn{2}{|l|}{ Education } \\
\hline College graduate & $1623(37.6 \%)$ \\
\hline High school graduate & 1705 (37.5\%) \\
\hline Middle school graduate & $549(8.7 \%)$ \\
\hline Elementary school graduate & $1179(16.3 \%)$ \\
\hline \multicolumn{2}{|l|}{ Meeting the physical activity guideline } \\
\hline Yes & $2374(51.0 \%)$ \\
\hline No & 2691 (49.0\%) \\
\hline
\end{tabular}

a significantly lower odds (OR $=0.54$; 95\% CI: $0.46-0.62)$ of meeting the physical activity guideline compared with younger adults.

We observed two significant interaction effects (i.e., income $\times$ age; education $\times$ age; $p<0.01)($ Table 3$)$. First, in unadjusted and adjusted models, the association between income and physical activity was moderated by age group (Table 3, Model 2; also shown in Fig. 2). Specifically, after adjusting for sociodemographic covariates, younger adults (age $<45$ years) in the moderate-low household income group had a $41 \%$ higher odds of meeting the physical activity guideline compared with younger adults in the upper household income group $(\mathrm{OR}=1.41,95 \% \mathrm{CI}=1.02-1.97 ; p=0.04)$. In contrast, among older adults (age $\geq 45$ years) the odds of meeting the physical activity guideline decreased as household income decreased. Specifically, compared with the upper household income group, older adults in moderate-low household income had a $16 \%$ decreased odds of meeting the physical activity guideline $(\mathrm{OR}=0.84,95 \% \mathrm{CI}=0.72-$ $0.96 ; p=0.01)$, after adjusting for sociodemographic covariates (Fig. 2).

The second significant interaction effect showed that the association between education and physical activity was moderated by age in the unadjusted model (Table 3, Model 2). Specifically, among younger adults (age $<45$ years), high school graduates had a $21 \%$ higher odds of meeting the physical activity guideline compared with college graduates $(\mathrm{OR}=1.21,95 \% \mathrm{CI}=0.97-1.51 ; p=0.08)$. By contrast, among older adults, high school graduates had $23 \%$ lower odds of meeting the physical activity guideline compared with college graduates $(\mathrm{OR}=0.77,95 \% \mathrm{CI}=$ $0.65-0.89 ; p=0.01)$. The moderating effect was no longer significant after adjusting for covariates $(p>0.05)$.

\section{Discussion}

In this nationally representative study, we observed that $51 \%$ of adults in South Korea were meeting the physical activity guideline recommended by WHO. This estimate, which was computed using IPAQ, is similar to those reported in the US (52\%) from Behavioral Risk Factor Surveillance System data [13] and lower than Japan (66.2\%), Singapore (66.9\%), and China (75.9\%) from WHO's estimation using the Global Physical Activity Questionnaire [36]. Altogether, these indicate that adults in South Korea may be physically inactive compared to other high-income countries in Asia. However, prior research has shown that IPAQ and the Global Physical Activity Questionnaire both tend to show a trend of overreporting, which in turn leads to an underestimation of physical inactivity prevalence [37-40]. In fact, a pooled analysis involving 358 population-based surveys with 1.9 million participants that adjusted for this over-report suggested a much lower prevalence $(27.5 \%)$ of meeting the guideline [41]. Taken together, given the variability in measures assessing physical activity, comparison between countries should be made with caution. Based on a more detailed analysis, this trend in South Korea varied by SES characteristics, such that low income and low educational attainment were associated with lower physical activity - thereby suggesting potential health consequences related to low physical activity levels in these low SES groups. To our knowledge, this is the first study 
Table 2 Habitual Physical Activity Level by Two SES Indicators; KNHANES VI $(n=5065)$

\begin{tabular}{|c|c|c|c|c|c|c|}
\hline Household income & Upper & Moderate & Moderate-low & Low & test statistics & $p$-value \\
\hline Meeting PA guideline & $54.7 \%$ & $52.8 \%$ & $50.0 \%$ & $41.1 \%$ & $F=34.10$ & $<0.001$ \\
\hline Not meeting PA guideline & $45.3 \%$ & $47.2 \%$ & $50.0 \%$ & $58.9 \%$ & & \\
\hline Education & $\begin{array}{l}\text { College graduate } \\
\text { and above }\end{array}$ & $\begin{array}{l}\text { High school } \\
\text { graduate }\end{array}$ & $\begin{array}{l}\text { Middle school } \\
\text { graduate }\end{array}$ & $\begin{array}{l}\text { Elementary school } \\
\text { graduate and below }\end{array}$ & & \\
\hline Meeting PA guideline & $55.7 \%$ & $55.7 \%$ & $43.2 \%$ & $33.5 \%$ & $F=7.20$ & $<0.001$ \\
\hline Not meeting PA guideline & $44.3 \%$ & $43.9 \%$ & $48.4 \%$ & $57.2 \%$ & & \\
\hline
\end{tabular}

to demonstrate such disparities in physical activity behavior among adults in South Korea.

The associations between SES and physical activity level were complex. Specifically, we observed that age moderated the association between income and physical activity, such that younger adults with moderate-low income levels were more physically active than younger adults with highincome levels. In contrast, physical activity levels of older adults showed a reverse trend, such that physical activity levels declined among lower income groups. A similar trend was observed with education, but only in the unadjusted model, which highlights the possibility that income is a more proximal correlate of physical activity behavior than education. The specific mechanisms explaining these associations remain unclear. The effect modification of the SES-physical activity association by age can be interpreted with emphasis on the South Korean context. In South Korea, more than $45 \%$ adults who are in poverty are over 65 years old, and this is the highest rate among the 38 OECD countries [42]. In addition, there may be a cohort effect such that the current elderly generations were alienated from the formal education system when they were school age, due to the Korean War is the 1950s. In light of this specific national context, the SES*age interaction patterns found here warrant examination in other settings to determine generalizability. However, there is compelling evidence of decline in muscle mass with increasing age [43], which in part accounts for lower physical activity among older adults. One potential explanation for the trend observed among younger adults could be that younger adults at high income levels live more sedentary lives due to their occupations (e.g., office work, managerial positions) as well as due to amenities (e.g., private transportation, larger home dwellings) that permit reduced levels of daily physical exertion, whereas younger adults with more modest income levels might have jobs or social contexts that require greater levels of daily activity (e.g., physically laborious jobs, walking/biking for transportation). The association between reduced physical activity and income among older adults might reflect a different social explanation-i.e., that older adults with higher income levels have access to resources and environments that encourage physical activity for recreation (e.g., gyms and sports clubs), whereas older adults with lower income levels lack these conditions that permit physical recreation. Further research is necessary to examine the specific factors that might explain these physical activity disparities in accordance with income and age in South Korea.

In light of the growing health disparities in Korea, these moderating effects warrant future investigation for hypothesis-driven social science research as well as evidenced-based policy implementation. For example, interventions that promote access to recreational activities (e.g., sports teams or clubs) might be effective at promoting physical activity for older adults with low income and education levels, who might lack these resources and for whom physical activity might not be seen as enjoyable or rewarding. In contrast, interventions that caution against sedentary lifestyles might be effective at promoting physical activity for high-income younger adults, for whom the demands of work and the use of modern amenities might render physical activity as a low priority.

More broadly, one of the major policy implications from this work is that South Korea should conduct regular surveillance of physical activity. This data could be used, in turn, by public health scientists and policy makers to identify correlates and determinants of physical activity that should be targeted via intervention, policy, and campaigns. Another policy implication is that KNHANES may consider adding the Global Physical Activity Questionnaire to the existing IPAQ so as to facilitate a valid between-country comparison.

There were several strengths of the present study. We examined nationally representative data using weighted analysis, which permits generalization of findings to South Korean adults. Therefore, the observations from the present study have high external validity. Moreover, the IPAQ is a well-validated, reliable prognostic indicator of clinical health outcomes that can effectively discriminate between populations [44-46]. Finally, while prior studies examined the association between SES and physical activity among adolescents [26], the elderly [27], and metabolic syndrome patients [28] in South Korea [26], this is the first study to examine the association among adults in South Korea and is an important step toward understanding population patterns associated with physical activity in this national context. 
Table 3 Weighted Logistic Regressions examining Associations between Socioeconomic Status and Age with Physical Activity; KNHANES, VI $(n=5065)$

\begin{tabular}{|c|c|c|c|c|c|c|}
\hline \multirow{2}{*}{ Model 1} & \multicolumn{3}{|c|}{ Unadjusted } & \multicolumn{3}{|c|}{ Fully Adjusted $^{a}$} \\
\hline & OR & $\mathrm{Cl}$ & p-value & Adjusted OR & $\mathrm{Cl}$ & $p$-value \\
\hline \multicolumn{7}{|l|}{ Household Income } \\
\hline Upper & 1.00 & $\mathrm{n} / \mathrm{a}$ & $\mathrm{n} / \mathrm{a}$ & 1.00 & $\mathrm{n} / \mathrm{a}$ & $\mathrm{n} / \mathrm{a}$ \\
\hline Moderate & 0.92 & $0.75-1.14$ & 0.47 & 1.01 & $0.80-1.29$ & 0.89 \\
\hline Moderate-low & 0.83 & $0.69-0.99$ & 0.04 & 1.08 & $0.87-1.35$ & 0.47 \\
\hline Low & 0.58 & $0.47-0.71$ & $<0.01$ & 0.80 & $0.59-1.10$ & 0.17 \\
\hline \multicolumn{7}{|l|}{ Education } \\
\hline College graduate & 1.00 & $\mathrm{n} / \mathrm{a}$ & $\mathrm{n} / \mathrm{a}$ & 1.00 & $\mathrm{n} / \mathrm{a}$ & $n / a$ \\
\hline High school graduate & 1.00 & $0.85-1.19$ & 0.99 & 1.12 & $0.91-1.36$ & 0.28 \\
\hline Middle school graduate & 0.61 & $0.48-0.77$ & $<0.01$ & 0.83 & $0.62-1.11$ & 0.21 \\
\hline Elementary school graduate & 0.40 & $0.33-0.49$ & $<0.01$ & 0.70 & $0.52-0.93$ & 0.02 \\
\hline \multicolumn{7}{|l|}{ Age } \\
\hline Younger (Age < 45) & 1.00 & $\mathrm{n} / \mathrm{a}$ & $\mathrm{n} / \mathrm{a}$ & 1.00 & $\mathrm{n} / \mathrm{a}$ & $\mathrm{n} / \mathrm{a}$ \\
\hline Older (Age $\geq 45$ ) & 0.54 & $0.46-0.62$ & $<0.01$ & 0.75 & $0.63-0.89$ & $<0.01$ \\
\hline Model 2: SES*Age Interaction & OR & $\mathrm{Cl}$ & p-value & Adjusted OR & $\mathrm{Cl}$ & $p$-value \\
\hline \multicolumn{7}{|l|}{ Household Income } \\
\hline Younger upper & 1 & $n / a$ & $\mathrm{n} / \mathrm{a}$ & 1.00 & $\mathrm{n} / \mathrm{a}$ & $n / a$ \\
\hline Younger moderate & 1.28 & $1.11-1.48$ & 0.08 & 1.15 & $0.84-1.58$ & 0.38 \\
\hline Younger moderate-low & 1.21 & $0.97-1.51$ & 0.79 & 1.41 & $1.02-1.97$ & 0.04 \\
\hline Younger low & 0.92 & $0.42-0.94$ & 0.3 & 1.10 & $0.57-2.13$ & 0.77 \\
\hline Older upper & 0.93 & $0.75-1.16$ & 0.55 & 1.22 & $0.90-1.66$ & 0.20 \\
\hline Older moderate & 0.67 & $0.49-0.91$ & 0.01 & 1.07 & $0.88-1.30$ & 0.19 \\
\hline Older moderate-low & 0.67 & $0.30-1.53$ & 0.34 & 1.02 & $0.88-1.18$ & 0.01 \\
\hline Older low & 0.76 & $0.24-2.43$ & 0.65 & 0.79 & $0.44-1.42$ & 0.15 \\
\hline \multicolumn{7}{|l|}{ Education } \\
\hline Younger college graduate & 1.00 & $\mathrm{n} / \mathrm{a}$ & $\mathrm{n} / \mathrm{a}$ & 1.00 & $\mathrm{n} / \mathrm{a}$ & $\mathrm{n} / \mathrm{a}$ \\
\hline Younger high school graduate & 1.21 & $0.97-1.51$ & 0.08 & 1.14 & $0.88-1.47$ & 0.34 \\
\hline Younger middle school graduate & 0.90 & $0.42-1.94$ & 0.79 & 1.59 & $0.63-3.98$ & 0.32 \\
\hline Younger elementary school graduate & 0.54 & $0.17-1.74$ & 0.30 & 0.76 & $0.19-3.07$ & 0.70 \\
\hline Older college graduate & 0.93 & $0.75-1.16$ & 0.55 & 1.08 & $0.82-1.42$ & 0.57 \\
\hline Older high school graduate & 0.77 & $0.65-0.89$ & 0.01 & 1.15 & $0.39-1.57$ & 0.73 \\
\hline Older middle school graduate & 0.57 & $0.20-1.59$ & 0.35 & 0.81 & $0.54-1.18$ & 0.13 \\
\hline Older elementary school graduate & 0.39 & $0.01-15.07$ & 0.65 & 0.73 & $0.01-45.6$ & 0.86 \\
\hline
\end{tabular}

a Models controlled for marital status, town type, dwelling type, perceived health, federal allowance support, and working- and sleeping-hours

Statistically significant data $(p<0.05)$ are presented in bold

There were also several limitations of the present study. We cannot infer the causal effect of SES on physical activity because we employed a cross-sectional design. Reverse causation is theoretically plausible, such that physical activity level may influence household income and academic achievements. However, this scenario seems unlikely considering existing empirical research and social science theory that highlights the effect of SES on health behavior in general [32, 47] and physical activity in particular [20-25], rather than the reverse pathway. There may be limitations to using household income for age-specific comparisons. For example, using household income assumes that all family members benefit equally from the household income, which may not be the case. Finally, physical activity was measured using a self-report questionnaire, which is susceptible to biases [48]. However, the Korean version of IPAQ has been shown to be valid and reliable [33, 34]. 


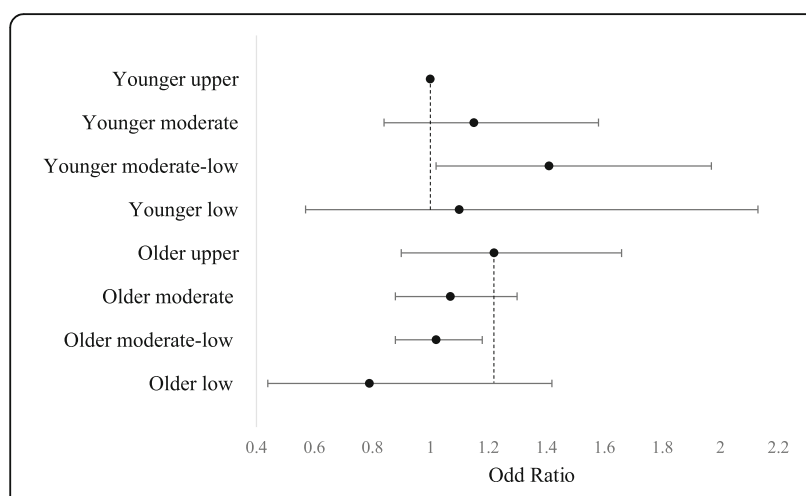

Fig. 2 Household Income and Odds of meeting the Physical Activity Guideline by Age, Adjusting for Covariates

\section{Conclusion}

Overall, roughly half of adults in South Korea are physically inactive, which indicates a high proportion of inactivity in comparison with adults in other high-income national settings. Consistent with growing research on socioeconomic disparities in Korea, the proportion meeting the WHO recommendation for physical activity-which is an important behavior that can promote overall health and wellbeing-was disproportionately higher among wealthy and educated adults in Korea. Future policies that aim to address physical activity-related health disparities in Korea must incorporate different patterns of physical activity in accordance with SES and age. Specifically, a more tailored intervention or policy approach may be required to address lower physical activity levels among younger adults with higher income and among older adults with lower income. A tailored approach among older adults with lower income appears to have particularly high public health relevance given the rapidly aging population in South Korea.

\section{Abbreviations}

IPAQ: International Physical Activity Questionnaire; KNHANES: Korea National Health and Nutrition Examination Survey; NCDs: Noncommunicable diseases; SES: Socioeconomic status; WHO: World Health Organization

\section{Acknowledgments}

The authors are thankful for Dr. Shira Dunsiger's feedback on statistical analyses.

\section{Authors' contributions}

All authors collaborated in designing the study. HHL performed the data analyses and drafted the manuscript, with AEP and DO providing critical revisions to the manuscript. All authors read and approved the final manuscript.

\section{Funding}

$\mathrm{NIH/NHLBI}$ (1F31HL140817) supported the time of Harold Lee to perform the data analyses and write the manuscript.

\section{Availability of data and materials}

The Korea National Health and Nutrition Examination Survey (KNHANES) data utilized for the present article is publicly available through the KNHANES website (http://knhanes.cdc.go.kr). The website is only available in Korean. In the website, click"

receive the data.

". Then, enter an email address to

\section{Ethics approval and consent to participate}

Ethical approval was not required because names, places, ID numbers, or additional identifiers associated with the study population were anonymized before analysis.

\section{Consent for publication}

Not applicable.

\section{Competing interests}

The authors declare that they have no competing interests.

\section{Author details}

'Department of Social and Behavioral Sciences, Harvard T.H. Chan School of Public Health, 401 Park Dr., 428F, Boston, MA 02115, USA. ²Department of Social and Behavioral Sciences, University of California San Francisco School of Nursing, 3333 California St., Suite 455, San Francisco, CA 94118, USA. ${ }^{3}$ Department of Behavioral and Social Sciences, Brown University School of Public Health, Box G-S121-3, Providence, RI 02912, USA.

Received: 3 September 2018 Accepted: 10 September 2019

Published online: 22 October 2019

\section{References}

1. Kuznets PW. An east Asian model of economic development: Japan, Taiwan, and South Korea. Econ Dev Cultural Change. 1988;36(S3):S11-43.

2. S-y B, K-k K. Educational inequality in South Korea: the widening socioeconomic gap in student achievement. In: Globalization, changing demographics, and educational challenges in East Asia. Bingley: Emerald Group Publishing Limited; 2010. p. 155-82.

3. Fields GS, Yoo G. Falling labor income inequality in Korea's economic growth: patterns and underlying causes. Rev Income Wealth. 2000;46(2):139-59.

4. Cha SH, Park HS, Cho HJ. Socioeconomic disparities in prevalence, treatment, and control of hypertension in middle-aged Koreans. J of Epidemiol. 2012;22(5):425-32

5. Hwang J, Shon C. Relationship between socioeconomic status and type 2 diabetes: results from Korea National Health and nutrition examination survey (KNHANES) 2010-2012. BMJ Open. 2014;4(8):e005710.

6. Kang JH, Jeong BG, Cho YG, Song HR, Kim KA. Socioeconomic costs of overweight and obesity in Korean adults. J of Korean Med Sci. 2011;26(12):1533-40.

7. Khang Y-H, Kim HR. Explaining socioeconomic inequality in mortality among south Koreans: an examination of multiple pathways in a nationally representative longitudinal study. Int J Epidemiol. 2005;34(3):630-7.

8. Park MJ, Yun KE, Lee GE, Cho HJ, Park HS. A cross-sectional study of socioeconomic status and the metabolic syndrome in Korean adults. Annals Epidemiol. 2007:17(4):320-6.

9. Lee I-M, Shiroma EJ, Lobelo F, Puska P, Blair SN, Katzmarzyk PT, et al. Effect of physical inactivity on major non-communicable diseases worldwide: an analysis of burden of disease and life expectancy. Lancet. 2012;380(9838):219-29.

10. World Health Organization. Global recommendations on physical activity for health. World Health Organization website [Available from: http://www.who. int/dietphysicalactivity/factsheet_adults/en/ ] Accessed 15 Aug 2018.

11. World Health Organization. Gelobal strategy on diet, physical activity and health: a framework to monitor and evaluate implementation. [Available from: http://www.who.int/dietphysicalactivity/Indicators\%20English.pdf ] Accessed 15 Aug 2018.

12. Hallal PC, Andersen LB, Bull FC, Guthold R, Haskell W, Ekelund U, et al. Global physical activity levels: surveillance progress, pitfalls, and prospects. Lancet. 2012;380(9838):247-57.

13. World Health Organization. Global health observatory data repository 2018 [Available from: http://www.who.int/gho/ncd/risk_factors/physical_activity/ en/] Accessed 15 Aug 2018.

14. Department of Health. Australia's physical activity and sedentary behaviour guidelines for adults (18-64 years) Australia: Department of Health; 2014 [Available from: http://www.abs.gov.au/ausstats/abs@.nsf/Lookp/4364.0.55. 004Chapter1002011-12]. Accessed 15 Aug 2018.

15. Niblett P. Statistics on Obesity, Physical Activity and Diet, England - 2016 United Kindom: Health and Social Care Information Centre; 2015 [Available from: http://www.hscic.gov.uk/pubs/sopad16]. Accessed 15 Aug 2018. 
16. Troiano RP, Berrigan D, Dodd KW, Masse LC, Tilert T, McDowell M. Physical activity in the United States measured by accelerometer. Med Sci Sports Exer. 2008;40(1):181.

17. OECD. Hours worked (indicator); 2019. https://doi.org/10.1787/47be1c78-en. [available from: ] accessed on 16 Apr 2019

18. McKnight-Eily LR, Eaton DK, Lowry R, Croft JB, Presley-Cantrell L, Perry GS. Relationships between hours of sleep and health-risk behaviors in US adolescent students. Prev Med. 2011:53(4-5):271-3.

19. Virtanen $M$, Ferrie JE, Gimeno D, Vahtera J, Elovainio M, Singh-Manoux A, et al. Long working hours and sleep disturbances: the Whitehall II prospective cohort study. Sleep. 2009;32(6):737-45

20. Armstrong T, Bauman AE, Davies J. Physical activity patterns of Australian adults: results of the 1999 National Physical Activity Survey: Australian Institute of Health and Welfare; 2000. [Available from: https://www.aihw.gov. au/reports/physical-activity/physical-activity-patterns-of-australian-adults/ contents/table-of-contents] Accessed 15 Aug 2018.

21. Bauman A, Bellew B, Booth M, Hahn A, Stoker L, Thomas M. Towards best practice for the promotion of physical activity in the areas of NSW. NSW health department, Centre for Disease Prevention and Health Promotion. 1996. [Available from: https://www.health.nsw.gov.au/phb/Documents/1 997-3.pdf]. Accessed 15 Aug 2018.

22. Cauley JA, Donfield SM, LaPorte RE, Warhaftig NE. Physical activity by socioeconomic status in two population based cohorts. Med Sci Sports Exer. 1991;23(3):343-51.

23. Droomers M, Schrijvers $C T$, Van de Mheen H, Mackenbach JP. Educational differences in leisure-time physical inactivity: a descriptive and explanatory study. Soc Sci Med. 1998;47(11):1665-76.

24. Giles-Corti B, Donovan RJ. Socioeconomic status differences in recreational physical activity levels and real and perceived access to a supportive physical environment. Prev Med. 2002;35(6):601-11.

25. Yen $I H$, Kaplan GA. Poverty area residence and changes in physical activity level: evidence from the Alameda County study. Am J of Public Health. 1998;88(11):1709-12

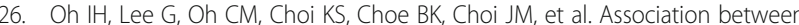
the physical activity of Korean adolescents and socioeconomic status. J Prev Med Public Health. 2009;42(5):305-14.

27. Lee $\mathrm{JH}$, Cho YT. Activity limitation and health behavior of the elderly in Seoul by socioeconomic level. Korean J of Geront. 2008;28(1):87-104.

28. Lee H, Kim BH. Physical activity disparities by socioeconomic status among metabolic syndrome patients: the fifth Korea National Health and nutrition examination survey. J Exer Rehabil. 2016;12(1):10-4

29. Luke A, Dugas LR, Durazo-Arvizu RA, Cao G, Cooper RS. Assessing physical activity and its relationship to cardiovascular risk factors: NHANES 20032006. BMC Public Health. 2011;11(1):1

30. Kim Y. The Korea National Health and nutrition examination survey (KNHANES): current status and challenges. Epidemiol Health. 2014;36: e2014002.

31. Kweon S, Kim Y, M-j J, Kim Y, Kim K, Choi S, et al. Data resource profile: the Korea national health and nutrition examination survey (KNHANES). Int J Epidemiol. 2014;43(1):69-77.

32. Adler NE, Boyce T, Chesney MA, Cohen S, Folkman S, Kahn RL, et al. Socioeconomic status and health: the challenge of the gradient. Am Psychol. 1994;49(1):15.

33. Chun MY. Validity and reliability of korean version of international physical activity questionnaire short form in the elderly. Korean J Fam Med. 2012;33(3):144-51.

34. Oh JY, Yang YJ, Kim BS, Kang JH. Validity and reliability of Korean version of international physical activity questionnaire (IPAQ) short form. J Korean Academy of Fam Med. 2007;28(7):532-41.

35. Team RC. R: a language and environment for statistical computing, $R$ software version 3.3. 1; 2016

36. Center for Disease Control and Prevention. Adult participation in aerobic and muscle-strengthening physical activities--United States, 2011. MMWR Morbidity and mortality weekly report. 2013;62(17):326 [Available from: https://www.cdc.gov/mmwr/preview/mmwrhtml/mm6217a2.htm?s_cid= mm6217a2_e] Accessed 15 Aug 2018.

37. Rzewnicki R, Vanden Auweele Y, De Bourdeaudhuij I. Addressing overreporting on the international physical activity questionnaire (IPAQ) telephone survey with a population sample. Public Health Nutr. 2003:6:299-305.

38. Hallal PC, Gomez LF, Parra DC, et al. Lessons learned after 10 years of IPAQ use in Brazil and Colombia. J Phys Act Health. 2010;7(suppl 2):S259-64.
39. Ekelund U, Sepp H, Brage S, et al. Criterion-related validity of the last 7-day, short form of the international physical activity questionnaire in Swedish adults. Public Health Nutr. 2006:9:258-65.

40. Ainsworth BE, Macera CA, Jones DA, et al. Comparison of the 2001 BRFSS and the IPAQ physical activity questionnaires. Med Sci Sports Exerc. 2006;38:1584-9244.

41. Guthold R, Stevens GA, Riley LM, Bull FC. Worldwide trends in insufficient physical activity from 2001 to 2016: a pooled analysis of 358 population-based surveys with 1.9 million participants. Lancet Glob Health. 2018;6(10):e1077-86.

42. OECD. Poverty rate (indicator); 2019. https://doi.org/10.1787/0fe1315d-en. [available from: ] 1 Accessed on Apr 2019

43. Ingram DK. Age-related decline in physical activity: generalization to nonhumans. Med Sci Sports Exer. 2000;32(9):1623-9.

44. Alomari MA, Keewan EF, Qhatan R, Amer A, Khabour OF, Maayah MF, et al. Blood pressure and circulatory relationships with physical activity level in young normotensive individuals: IPAQ validity and reliability considerations. Clinic Experimental Hypertens. 2011;33(5):345-53.

45. Lee PH, Macfarlane DJ, Lam T, Stewart SM. Validity of the international physical activity questionnaire short form (IPAQ-SF): a systematic review. Int J of Behav Nutr Phys Act. 2011:8(1):115

46. Teo K, Lear S, Islam S, Mony P, Dehghan M, Li W, et al. Prevalence of a healthy lifestyle among individuals with cardiovascular disease in high-, middle-and low-income countries: the prospective urban rural epidemiology (PURE) study. JAMA. 2013;309(15):1613-21.

47. Lynch JW, Kaplan GA, Salonen JT. Why do poor people behave poorly? Variation in adult health behaviours and psychosocial characteristics by stages of the socioeconomic lifecourse. Soc Sci Med. 1997:44(6):809-19.

48. Paulhus DL. Measurement and control of response bias. In: Robinson JP, Shaver PR, Wrightsman LS, editors. Measures of personality and social psychological attitudes. San Diego: Academic Press; 1991. p. 17-59.

\section{Publisher's Note}

Springer Nature remains neutral with regard to jurisdictional claims in published maps and institutional affiliations.
Ready to submit your research? Choose BMC and benefit from:

- fast, convenient online submission

- thorough peer review by experienced researchers in your field

- rapid publication on acceptance

- support for research data, including large and complex data types

- gold Open Access which fosters wider collaboration and increased citations

- maximum visibility for your research: over $100 \mathrm{M}$ website views per year

At $\mathrm{BMC}$, research is always in progress.

Learn more biomedcentral.com/submission 